\title{
Do Squat Exercises With Weight Shift During Pregnancy Improve Postural Control?
}

\author{
Kaname Takeda ${ }^{1^{* \mathbb{C}}}$, Hiromi Yoshikata ${ }^{2}$, Masumi Imura ${ }^{3}$
}

\begin{abstract}
Objectives: The present study aimed to investigate the effect of squat exercises with weight shift on muscle strength and postural control during pregnancy.

Materials and Methods: The squat group comprised 21 pregnant women in their 20s and 30s with no exercise habits while the control group consisted of 20 randomly selected pregnant women in their 20 s and 30 s with no exercise habits. The squat group was instructed to perform two sets of daily squat exercises with weight shift in the anterior, posterior, right, and left directions, with 10 repetitions counted as one set, for 10 weeks. The stabilometer was used to measure postural control ability in the second and third trimesters. Meanwhile, muscular strength was taken as the toe grip force and quadriceps muscle strength before and after the intervention.

Results: In the squat group, significant increases were observed in the index of postural stability and the stability area. Conversely, however, significant decreases were detected in the average rectangular area. In addition, in the intervention group, significant declines were observed in the rectangular area in the posterior, right, and left directions. However, a considerable increase in muscular strength was only seen after the intervention in toe grip force.

Conclusions: Based on the results, it was concluded that everyday squat exercises which were performed during pregnancy improved postural control. The improvement of postural control during squat movements was thought to have a greater impact on plantar sensation than on muscle strength. These findings suggest that squat exercises with weight shift may promote fall prevention.

Keywords: Pregnant women, Postural control, Squat
\end{abstract}

\section{Introduction}

According to previous studies, falls are reported by $18 \%-25 \%$ of women during pregnancy (1-3). Although temporary, reduced postural control ability such as postural sway and displacement of the anterior-posterior center of gravity is often detected in pregnancy (2-4). A survey of fall situations in Japan reported that falls due to wobble during anti-weight/gravity actions like descending the stairs and squatting/rising from sitting increased during pregnancy (5). Several factors have been identified to reduce postural control ability in pregnancy including increased spinal lordosis, reduced strength of the abdominal muscles, and loosening of the major joints (2). Previous studies reported that pregnant women who experience a fall show increased rectangular areas in the posterior, lateral direction and decreased stability limits from the second trimester, and a sharp increase in abdominal circumference from the second to third trimester (6). The lack of awareness of sudden changes in abdominal circumference from the second to the third trimester was also considered as another factor of falling (6).

These reports suggest a need to incorporate exercises that improve awareness in addition to enlightenment and guidance regarding the prevention of falls during pregnancy. During this period, the load to the lower limbs increases day by day in antigravity actions such as standing up and sitting (7). Therefore, antigravitational exercise is essential to improve postural control during pregnancy and promote muscle activity that supports self-weight while being conscious of wobble owing to the weight shift in the front, back, left, and right directions. In addition, these exercises should be performed every day to allow changes in weight and the displacement of the center of gravity during the course of pregnancy so that this period is perceived more easily by the women.

The squat is listed as an antigravitational exercise. The squat movement includes crouching and rising elements that are necessary for activities of daily living. When squat exercises are performed with a weight shift in the anterior, posterior, right, and left directions, the exercise load changes as well. In previous studies, higher muscle activity was observed in the quadriceps femoris and tibialis anterior muscles while in the squat position the center of gravity line was displaced in the posterior direction $(8,9)$. When squatting with anterior weight shift,

Received 15 June 2018, Accepted 9 September 2018, Available online 2 October 2018

${ }^{1}$ Division of Physical Therapy, Department of Rehabilitation Sciences, Faculty of Allied Health Sciences, Kansai University of Welfare Sciences, Osaka, Japan. ${ }^{2}$ Yoshikata Obstetrics and Gynecology, 2430 Kozukuecho, Kohoku Ward, Yokohama, Kanagawa 222-0036, Japan. ${ }^{3}$ Japanese Red Cross College of Nursing, Graduate School of Nursing, International Health Care and Midwifery, 4-1-3 Hiroo, Shibuya-ku, Tokyo 150-0012, Japan. .

*Corresponding Author: Kaname Takeda, Tel: +81729780088, Email: takeda@tamateyama.ac.jp 
hip joint extension and ankle flexion muscle group activity are required. Conversely, when squatting with posterior weight shift, knee extension activity is needed. Moreover, while squatting with weight shift in the lateral direction, hip abductor muscle group activity is necessary. In addition, squatting with weight shift entails postural control ability that does not deviate from the center of pressure in terms of stability limits. Increases in body weight accompanying pregnancy become a type of resistance exercise, and daily exercising to adapt to positional changes in the center of gravity can help rebuild the body schema. However, to the best knowledge of the authors, no prior studies have examined exercise for the purpose of fall prevention and postural control maintenance.

Mental and physical exercise guidance and conditioning are therefore considered important for fall prevention during the pregnancy. Accordingly, the purpose of this study was to explore the effect of squat exercises with weight shift on postural control and muscle strength.

\section{Materials and Methods}

The participants in the squat intervention group included 21 healthy pregnant women in their 20s and 30s. All the participants understood the purpose and content of the study and consulted the Obstetrics and Gynecology Department before providing their written informed consent in order to participate. The exclusion criteria were having a history of lower limb or lower back injuries or regular exercise habits. The participants of the squat group were given both oral and written instructions regarding the intervention method, which involved performing two sets of daily squat exercises with weight shift in the anterior, posterior, right, and left directions including 10 repetitions counted as one set, for 10 weeks.

Either the starting position of the limbs or how to apply the load during the squat exercises were in accordance with the locomotor training squat method targeting the elderly advocated by the Japanese Orthopedic Association (10). The starting position of the upper limbs was with the arms slightly wider than shoulder width, and that of the lower limbs included the feet apart with the toes facing outward at about a $30^{\circ}$ angle. Squats were performed at a steady 1 -second pace while breathing regularly and maintaining a knee flexion angle that ensured no burden (less than $90^{\circ}$ ). Regarding the weight shift method, the participants were taught to squat while shifting their weight to the toes as the anterior movement, and then to continue squatting whereas moving the weight sequentially to the heel, the right foot, and the left foot, and subsequently to repeat these steps again from the beginning.

During the intervention, participants in poor physical conditions could stop at any time based on their own judgment. The Cybozu Live smartphone application (Cybozu Co., Ltd., Tokyo, Japan) was used to record a daily implementation report. If there was no report logged by $10 \mathrm{PM}$, the app notified the participant for confirmation in order to prevent them from forgetting to enter their data.

Periodic medical examinations were conducted by a gynecologist before performing the measurements after confirming the participant's physical condition.

For quantitative evaluation of postural control ability, two stabilometers (JK-101 II, Unimec Co., Tokyo, Japan) and a personal computer (Dynabook B553, TOSHIBA Co., Ltd., Tokyo, Japan) were employed. As a measurement task, each participant was instructed to maintain a stationary standing position on the two stabilometers while barefoot, with the medial malleoli $100 \mathrm{~mm}$ apart. The participants were instructed to signal "yes" when they considered body sway stable, and then to maintain the same posture for another 10 seconds. They were then asked to maintain a stable posture while their center of gravity was moving as far as possible in the anterior direction, in the same manner as that while standing. Thereafter, the center of gravity was moved in the posterior direction in the same manner, and then to the right and left. In each case, data were extracted for 10 seconds from the time the participant signaled to move the center of gravity in each position. These tasks were conducted twice during each measurement session.

The parameters analyzed in the second and third trimesters were as follows: 1) Index of postural stability (IPS), 2) Average rectangular area in each position, 3) Stability limits, and 4) Rectangular area for posture held for 10 seconds in the anterior, posterior, right, and left directions. The IPS measures stability using stable and rectangular areas and is strongly correlated with the Berg Balance Scale (11). This index is calculated by summing the average rectangular area in each position and the area of the stability limits, divided by the average rectangular area to obtain the logarithmic value (11). Stability limits are obtained by multiplying the movement of the center of gravity in the anterior-posterior and lateral directions as well.

The physical characteristics of the participants were obtained in the interviews and based on the results, periodic medical examinations were performed in the second or third trimester. The interview items were related to the height, weight at the time of measurement, and abdominal circumference. All the measurements were duplicated in the second and the third trimesters.

To assess the effect of squat exercises on the muscles, the toe grip force acting was measured on postural control in the anterior direction and the muscle force of the quadriceps, which is an antigravity muscle. Toe grip force was calculated using a toe grip dynamometer (TKK3364, Takei Scientific Instruments, Niigata, Japan). The participants were instructed to maintain $90^{\circ}$ knee flexion and $0^{\circ}$ ankle dorsiflexion while sitting. The muscular strength of the toe in each participant was then measured by getting them to use their toes to grasp the dynamometer with maximum voluntary effort (Figure 1A). 
The strength of the quadriceps muscle was determined applying a handheld dynamometer (HHD) ( $\mu$ TAS MF01, Anima Co., Ltd., Tokyo, Japan) according to the method employed by Kato et al (12). The starting limb position was seated with the knee joint at $90^{\circ}$ flexion. The maximum isometric muscle force of the quadriceps femoris was calculated twice on the right and left legs with the sensor part of the HHD placed at the distal part of the lower leg and fixed to the strut of the treatment bed with a belt (Figure 1B). Muscle force was duplicated in both legs, and the mean of the maximum values was adopted.

The control group comprised 20 randomly selected pregnant women in their 20s and 30s. Measurement data in this group were selected applying a random number generator. All the data were used only after obtaining approval by telephone from those who did not have regular exercise habits.

This study was implemented following the approval of the Ethics Committee at the Kansai University of Welfare Science (under the approval number 16-10). The SPSS (statistical package for the social sciences) software, version 24 (IBM, Tokyo, Japan) was used for statistical analysis including: 1) a comparison of physical characteristics between the squat intervention and control groups, and of muscle strength in the squat intervention group before and after the intervention and 2) a comparison of postural control parameters and time points between both groups. The Wilcoxon signed-rank sum test was used to analyze $1)$ the level of significance $(P<0.05)$. Moreover, two-way repeated-measures analysis of variance (ANOVA) was employed to examine 2$)$ the level of significance $(P<0.05)$ through using the Bonferroni correction for further analysis.

\section{Results}

The physical characteristics of each group are shown in Table 1. After excluding the dropouts and those who chose not to participate because of a poor physical condition, 20 women participated in the squat intervention group. No significant differences were observed in either group respecting age, height, weight, or abdominal circumference.

Changes in the IPS, representative and stability areas, and the rectangular area in each direction for both groups are shown in Table 2. Based on the results of two-way ANOVA, an interaction was observed for the IPS (F (1, $78)=69.26, P<0.05)$. In a subsequent simple primary effect test, the IPS was significantly higher in the control group in the second trimester $(P=0.02)$ and in the squat intervention group in the third trimester $(P=0.01)$. In addition, the IPS decreased significantly (4.5\%) with pregnancy progression in the control group $(P=0.01)$ while it increased significantly $(6.1 \%)$ in the squat intervention group $(P=0.01)$. Besides, an interaction was observed in the mean rectangular area $(\mathrm{F}(1,78)=$

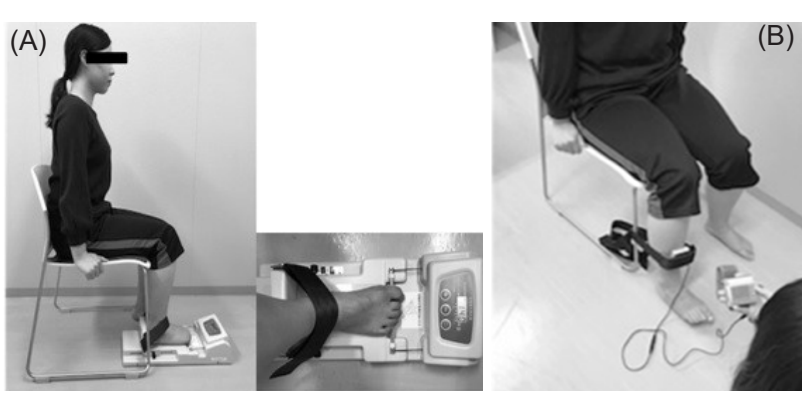

Figure 1. (A) Measurement method of toe grip force. Subjects are instructed to grasp the instrument bar with the maximum force with the toes. (B) Measurement method of knee extension muscle. Fixed to the legs of the chair with a belt not stretchable. Instructs to knee extension to tension the belt. Knee extension strength is extracted from the sensor.

43.70, $P<0.05)$. In a subsequent simple main effect test, no significant difference was found between the groups in the second trimester $(P=0.49)$. However, the mean area in the control group was significantly higher in the third trimester $(P=0.01)$. Furthermore, in the control group, the mean area enhanced significantly (26\%) with pregnancy progression $(P=0.01)$ while decreasing significantly $(18 \%)$ in the squat intervention group $(P=0.01)$. An interaction was observed in the stability limits $(\mathrm{F}(1,78)$ $=4.35, P<0.05)$ as well. In addition, in a subsequent simple primary effect test, significantly larger stability limits were detected in the control group in the second trimester $(P=0.01)$. Nonetheless, there were no significant differences in either group in the third trimester $(P=0.37)$. Moreover, although no significant changes were seen in the control group $(P=0.77)$, significant increases $(11 \%)$ were found in the squat intervention group $(P=0.01)$.

Despite these changes in the rectangular area, there were no interactions in timing and intervention, main effect, the standing position rectangular area, and the anterior rectangular area. Nevertheless, an interaction was observed in the posterior rectangular area $(\mathrm{F}(1,78)$

Table 1. Comparison of Physical Characteristics Between Control and Squat Groups

\begin{tabular}{lccc}
\hline & Control Group & Squat Group & $P$ Value \\
\hline Age & $32.3 \pm 3.2$ & $31.7 \pm 3.4$ & 0.92 \\
Height $(\mathrm{cm})$ & $159.7 \pm 5.3$ & $159.3 \pm 4.4$ & 0.89 \\
BMI & & & \\
2nd trimester & 21.8 & 22.2 & 0.30 \\
3rd trimester & 23.0 & 23.5 & 0.26 \\
Weight (kg) & & & \\
2nd trimester & $55.6 \pm 6.1$ & $56.5 \pm 6.6$ & 0.36 \\
3rd trimester & $58.7 \pm 5.6$ & $59.7 \pm 6.9$ & 0.32 \\
Abdominal circumference $(\mathrm{cm})$ & & \\
2nd trimester & $86.6 \pm 5.2$ & $86.2 \pm 4.6$ & 0.79 \\
3rd trimester & $92.1 \pm 4.6$ & $91.2 \pm 9.6$ & 0.47 \\
\hline *P $<.05$. & & &
\end{tabular}


Table 2. Comparison of Posture Control Abilities Between Control and Squat Groups

\begin{tabular}{|c|c|c|c|c|c|}
\hline & 2nd Trimester & 3rd Trimester & Time Main Effect & Intervention & Interaction \\
\hline IPS & & & $13.0^{*}$ & $1.54^{*}$ & $69.26^{*}$ \\
\hline Control group & $2.15 \pm 0.14$ & $2.05 \pm 0.17$ & & & \\
\hline Squat group & $2.05 \pm 0.17$ & $2.18 \pm 0.14$ & & & \\
\hline Average Rectangle area (mm2) & & & $6.19^{*}$ & $8.45^{*}$ & $43.70^{*}$ \\
\hline Control group & $153.10 \pm 65.15$ & $193.63 \pm 76.09$ & & & \\
\hline Squat group & $164.13 \pm 63.13$ & $135.00 \pm 44.40$ & & & \\
\hline Stability limits $\left(\mathrm{mm}^{2}\right)$ & & & $13.19^{*}$ & $5.04^{*}$ & $4.35^{*}$ \\
\hline Control group & $20076.32 \pm 4382.09$ & $20243.95 \pm 3636.21$ & & & \\
\hline Squat group & $17615.93 \pm 4037.59$ & $19516.57 \pm 3943.49$ & & & \\
\hline Rectangular Statistic area $\left(\mathrm{mm}^{2}\right)$ standing & & & 2.05 & 2.57 & 0.00 \\
\hline Control group & $86.32 \pm 35.98$ & $99.37 \pm 41.79$ & & & \\
\hline Squat group & $87.68 \pm 47.15$ & $89.85 \pm 49.70$ & & & \\
\hline Front & & & 0.01 & 0.33 & 0.30 \\
\hline Control group & $136.51 \pm 74.80$ & $153.20 \pm 85.49$ & & & \\
\hline Squat group & $126.85 \pm 81.90$ & $114.38 \pm 53.23$ & & & \\
\hline Back & & & 0.75 & $8.47^{*}$ & $17.68^{*}$ \\
\hline Control group & $191.90 \pm 98.72$ & $250.18 \pm 161.45$ & & & \\
\hline Squat group & $208.03 \pm 136.18$ & $151.61 \pm 68.94$ & & & \\
\hline Right & & & $5.60^{*}$ & $8.04^{*}$ & $13.68^{*}$ \\
\hline Control group & $181.29 \pm 106.47$ & $232.61 \pm 122.24$ & & & \\
\hline Squat group & $2013.28 \pm 120.80$ & $160.81 \pm 68.28$ & & & \\
\hline Left & & & $5.78^{*}$ & $13.63^{*}$ & $22.47^{*}$ \\
\hline Control group & $169.50 \pm 119.41$ & $232.78 \pm 127.00$ & & & \\
\hline Squat group & $184.80 \pm 78.16$ & $158.33 \pm 78.60$ & & & \\
\hline
\end{tabular}

Note. IPS: Index of postural stability; Control group ( $n=20)$; Squat group $(n=20) ;{ }^{*} p<0.05$.

$=17.68, P<0.05)$. Meanwhile, in the subsequent simple main effect test, no significant differences were found in the rectangular area in the second trimester $(P=0.60)$. Nonetheless, it was significantly smaller in the squat intervention group in the third trimester $(P=0.01)$. In the control group, there was a significant increase $(30 \%)$ in the rectangular area with pregnancy progression $(P=0.01)$ whereas it decreased significantly $(27 \%)$ in the squat intervention group $(P=0.01)$. Besides, an interaction was observed in the right rectangular area $(F(1,78)=13.68$, $P<0.05)$. Moreover, in the subsequent simple main effect test, no significant differences were seen between the groups in the second trimester $(P=0.25)$. However, it was significantly smaller in the squat intervention group in the third trimester $(P=0.01)$. In addition, a significant increase $(28 \%)$ whereas a significant decrease $(24 \%)$ were observed in the control $(P=0.01)$ and squat intervention $(P=0.02)$ groups with pregnancy progression, respectively. An interaction was also observed in the left rectangular area $(\mathrm{F}(1,78)=22.47, P<0.05)$. Furthermore, in the subsequent simple main effect test, no significant differences were seen between the groups in the second trimester $(P=0.43)$. However, it was significantly smaller in the squat intervention group in the third trimester $(P$ $=0.01)$. Moreover, it was significantly larger $(37 \%)$ in the control group $(P=0.01)$ while being significantly smaller $(14 \%)$ in the squat intervention group $(P=0.01)$ with pregnancy progression.

Changes in muscle force before and after the intervention in the squat intervention group are shown in Table 3. Although toe grip force increased significantly after the squat intervention $(P<0.05)$, no significant differences were found in the quadriceps femoris.

\section{Discussion}

The current study sought to investigate the effect of squat exercises with weight shift on muscle strength and postural control during pregnancy.

The IPS and stability area were higher in the second trimester in the control group than in the squat intervention group. In addition, the control group had better balance ability as compared to the squat intervention group in the second trimester.

Likewise, in the squat intervention group, both the IPS 
Table 3. Comparison of Muscle Strength Before and After Intervention

\begin{tabular}{llll}
\hline & Before & After & $P$ Value \\
\hline Foot grip strength $(\mathrm{kg})$ & $6.51 \pm 2.47$ & $8.18 \pm 2.67$ & $0.00^{*}$ \\
Quadriceps femoris muscle $(\mathrm{N})$ & $15.10 \pm 5.29$ & $14.87 \pm 5.79$ & 0.80 \\
\hline$* P<0.05$ & & &
\end{tabular}

and stability area increased with pregnancy progression and were significantly higher compared with the control group in the third trimester. Conversely, the mean rectangular area decreased significantly in the squat intervention group and was considerably smaller in the third trimester compared with that of the control group. These findings suggest that squat exercises improved postural control. Conversely, however, in the control group, the IPS decreased with pregnancy progression and was smaller in the third trimester. These findings indicated that a lack of exercise in normal pregnancies led to a decrease in postural control ability.

In the present study, the IPS was used to evaluate postural control. The IPS has reportedly been applied in cases of the neurological medical disease, cerebrovascular disorders, and cardiovascular disease, and also providing elderly support treatment. For example, in a study, a 67year old man and a 71-year old woman were found to have IPSs of 1.90 and 1.73, respectively, which both were lower than 2.00 (13). In the control group, the IPS was 2.15 in the second trimester decreasing to 2.05 in the third trimester. This means that at the end of a normal pregnancy, the IPS decreased to a level similar to that in the elderly. Conversely, the IPS increased with pregnancy progression in the squat intervention group and was equal to 2.17 at the end of the pregnancy. The IPS value can thus be interpreted as being larger than that of healthy people aged 21-54 years, suggesting improved postural control ability.

In the squat intervention group, the rectangular area in the posterior, right, and left directions decreased after the intervention. In the down phase of the squat, flexion of the hip joint, knee joint, ankle joint, and trunk must have been coordinated so that the center of gravity did not deviate from the stability area. It is considered that during pregnancy, the center of gravity line was displaced posteriorly and likely to deviate from the stability area. Squatting with a posterior weight shift so as not to deviate from the center of gravity was necessary to achieve control by proprioception and muscle force at the ankle dorsiflexors.

The intervention in this study was involved performing two sets of 10 repetitions of squat exercises per day. After the intervention, toe grip strength rather than the quadriceps muscle strength increased significantly. It seems that the number of occurrences ( 4 to 6 times per direction) was not sufficient for the muscle activity until the muscle strength of the quadriceps muscle increased. Continuous information from the sensory system regarding changes in the position of the body's center of gravity was important for maintaining the balance during standing. This information was reportedly obtained from the contact area between the toes, the sole, and the floor (14). Furthermore, squat exercises are found to stimulate a proprioceptive sensation and improve coordination in the lower limbs (8). Interventions that stimulate plantar sensation are thought to activate the mechanoreceptor of the plantar, which increases somatosensory information and improves balance ability (15-17). Therefore, squat exercises accompanied by changes in the center of gravity were thought to stimulate plantar sensation, which led to decreases in the posterior, right, and left rectangular areas in the squat intervention group.

Regarding the variation in both groups in static standing and the anterior rectangular area, in a previous study, body sway was reported to increase in the anteriorposterior direction during static standing in the third trimester $(4,5)$. However, foot width was not specified, especially in the static standing position. In the present study, foot width was measured while standing with feet $100 \mathrm{~mm}$ apart (2). The reason for the change in the rectangular area at standing was not identified, but it was likely because foot width in this study was believed to be set wider than that in the prior research. It seems that there may have been no changes owing to pregnancy progression. Since, the task of the squat intervention group was to perform squat exercises with a displaced center of gravity. Meanwhile, squatting in the normal standing position was not included as a condition. Therefore, it was considered that there was no change in the rectangular area of standing in the squat intervention group. The anterior rectangular area was measured while the participant moved the center of gravity line as far forward as possible holding the sit. Takeda et al. reported that compared with non-pregnant women, the posterior component of the floor reaction force in pregnant women decreased at the maximum functional reach test (FRT) with pregnancy progression. Conversely, the vertical component increased (18). This shows that non-pregnant women controlled their anterior postural attitude in such a way that the floor surface was pushed posterior whereas pregnant women seemed to have hardly any force to push the floor posterior. In other words, it can be inferred that anterior movement tasks did not involve postural control with trunk flexion during pregnancy but rather moved the center of gravity horizontally forward with trunk extension and ankle dorsiflexion. Normally, in forwarding the weight shift tasks, the toe grip force is said to be related to stability in the standing position and the forward movement of the center of gravity $(19,20)$. Although the toe grip force increased in the squat intervention group after the intervention, the forward mass movement caused by the trunk extension during early gestation and ankle dorsiflexion may have not resulted in a load on the 
forefoot. It is supposed that despite the increase in the toe grip force in the squat intervention group, the front rectangular area did not change due to postural control because the forefoot was not loaded in the forward center of gravity task.

\section{Conclusions}

In the squat intervention group, decreases were observed in the rectangular area in the posterior and lateral directions. Meanwhile, there were some increases in the stability limits, resulting in postural control ability similar to that in the elderly level. This intervention effect was not thought to be due to muscle enhancement but rather to the maintenance of proprioception. Maintaining proprioception may have prevented the deviation of the body schema promoting the prevention of falls. The risks of inappropriate and over-exercise predominantly affect the fetus (21). It is considered desirable to conduct such movements under strict medical supervision. It was suggested that the use of a smart-phone application would encourage regular exercise and enable a better physical condition management. The results of this study indicated that squat exercises with weight shifting are effective for maintaining postural control, which in turn leads to a reduced risk of falls.

This study did have some limitations which need to be acknowledged. The number of people in the intervention group was small. Therefore, the results may be generalized with caution.

Besides, the muscles which were examined to assess the effect of the intervention were localized. As a result, other body muscles are required to be investigated in order to evaluate the impact of such interventions.

In addition, the effect of the intervention on proprioception remained unclear. In the future, additional muscle groups in a larger sample should be investigated, and proprioception should be evaluated both before and after the intervention.

\section{Conflict of Interests}

Authors declare that they have no conflict of interests.

\section{Ethical Issues}

The ethical committee of Kansai University of Welfare Sciences approved the study(Approval number 16-10).

\section{Financial Support}

This research was conducted with the aid of a MEXT 2011 research grant (\#23593327).

\section{Acknowledgments}

The authors wish to thank all the pregnant participants and the instructors in the Departments of Midwifery and Obstetrics and Gynecology for their cooperation. This research was conducted in part thanks to aMEXT 2011 research grant (under the issue number 23593327).

\section{References}

1. Dunning K, LeMasters G, Levin L, Bhattacharya A, Alterman T, Lordo K. Falls in workers during pregnancy: risk factors, job hazards, and high risk occupations. Am J Ind Med. 2003;44(6):664-672. doi:10.1002/ajim.10318

2. Butler EE, Colon I, Druzin ML, Rose J. Postural equilibrium during pregnancy: decreased stability with an increased reliance on visual cues. Am J Obstet Gynecol. 2006;195(4):1104-1108. doi:10.1016/j.ajog.2006.06.015

3. Jang J, Hsiao KT, Hsiao-Wecksler ET. Balance (perceived and actual) and preferred stance width during pregnancy. Clin Biomech (Bristol, Avon). 2008;23(4):468-476. doi:10.1016/j.clinbiomech.2007.11.011

4. Takeda K, Shimizu K, Imura M. Change of stability limits in the third trimester. Journal of Human Life Engineering. 2014;15(1):58-64.

5. Takeda K, Imura M. Survey of falls in pregnant women. Japanese Journal of Maternal Health. 2016;56(4):591-598.

6. Takeda K, Yosikata H, Imura M. Changes in posture control of women that fall during pregnancy. Int J Womens Health Reprod Sci. 2018;6(3):255-262. doi:10.15296/ijwhr.2018.43

7. Takeda K, Katuhira J, Takano A. Examination of the intervention effect of sit-to-stand in the third trimester. Rigakuryoho Kagaku. 2012;27:73-76. doi:10.1589/rika.2773. [Japanese].

8. Ikezoe T, Ichihashi N, Morinaga T. Electromyographic analysis of the lower extremities during squatting: effects of changes in sagittal plane center of foot pressure on muscle activity. J Jpn Phys Ther Assoc. 2003;30(1):8-13. [Japanese].

9. Shimizu Y, Sunaga R, Usa H, et al. Kinematic analysis of the lower limbs in different squatting conditions. The Journal of Japan Academy of Health Sciences. 2016;18(4):200-209. [Japanese].

10. The Japanese Orthopedic Association. Squat, locomotive syndrome clinical guide 2010. Bunkodo, Tokyo; 2010:96-8. [Japanese].

11. Mochizuki H. The relationship between the perceived postural stability and indices of balance ability measured by posturography. Bunkyo Journal of Health Science Technology. 2009;(2):55-60. [Japanese].

12. Kato M, Yamazaki Y, Hiiragi Y. Measurements of isometric knee extension force with a hand-held dynamometerEffect for interrater reliability using fixing-belt. Sogo Rehabilitation. 2001;29(11):1047-1050. doi:10.11477/ mf.1552109624. [Japanese].

13. Mochizuki H, Mineshima T. Reliability and validity of the index of postural stability using forceplates. J Jpn Phys Ther Assoc. 2000;27(6):199-203. [Japanese].

14. Tanaka T, Maeda Y. Dynamic standing balance ability accompanying sensory disturbance of the elderly. Journal of the Society of Biomechanisms. 2015;39(4):205-210. doi:10.3951/sobim.39.205.

15. Preszner-Domjan A, Nagy E, Sziver E, Feher-Kiss A, Horvath G, Kranicz J. When does mechanical plantar stimulation promote sensory re-weighing: standing on a firm or compliant surface? Eur J Appl Physiol. 2012;112(8):2979-2987. doi:10.1007/s00421-011-2277-5

16. Ohsugi H, Motozuka T, Sakuma T, Yokoyama S, Murata 
S. Effect of the mechanical plantar stimulation for plantar cutaneous sensitivity and the toe gripping strength. Japanese Journal of Health Promotion and Physical Therapy. 2013;3(3):129-133. doi:10.9759/hppt.3.129

17. Kamei S, Kamei T, Iwatani M, et al. Effects of foot sole stimulation on body sway. Bulletin of Aino Gakuin. 2006;20:37-40.

18. Takeda K. Changes in the postural strategy in pregnancy. J Jpn Phys Ther Assoc. 2014;41(2):1148.

19. Fujiwara K, Ikegami H, Okada M, Koyama Y. Contribution of age and muscle strength of lower limbs to steadiness and stability in standing posture. Journal of the Anthropological Society of Nippon. 1982;90(4):385-399. doi:10.1537/ ase1911.90.385

20. Handa S, Horiuchi K, Aoki K. A study on the measurement of toes grasping strength and effect of standing postural control. Jpn J Ergon. 2004;40(3):139-147. [Japanese].

21. American Physical Therapy Association: Perinatal Exercise Guideline. Section on Obstetrics and Gynecology, Alexandria, VA: 1986.

(c) 2019 The Author (s); This is an open-access article distributed under the terms of the Creative Commons Attribution License (http://creativecommons.org/licenses/by/4.0), which permits unrestricted use, distribution, and reproduction in any medium, provided the original work is properly cited. 